\title{
Polarized optical feedback from an extremely short external cavity for controlling and stabilizing the polarization of vertical cavity surface emitting lasers
}

Cite as: Appl. Phys. Lett. 90, 121104 (2007); https://doi.org/10.1063/1.2714301

Submitted: 26 January 2007 . Accepted: 12 February 2007 . Published Online: 19 March 2007

Mikel Arizaleta Arteaga, Olivier Parriaux, Manuel López-Amo, Hugo Thienpont, and Krassimir Panajotov

\section{ARTICLES YOU MAY BE INTERESTED IN}

Single-fundamental-mode photonic-crystal vertical-cavity surface-emitting lasers

Applied Physics Letters 80, 3901 (2002); https://doi.org/10.1063/1.1481984

Tailoring light polarization in vertical cavity surface emitting lasers by isotropic optical feedback from an extremely short external cavity

Applied Physics Letters 89, 091102 (2006); https://doi.org/10.1063/1.2339040

Nonlinear dynamics accompanying polarization switching in vertical-cavity surface-emitting lasers with orthogonal optical injection

Applied Physics Letters 88, 101106 (2006); https://doi.org/10.1063/1.2181649

This article may be downloaded for personal use only. Any other use requires prior permission of the author and AIP Publishing

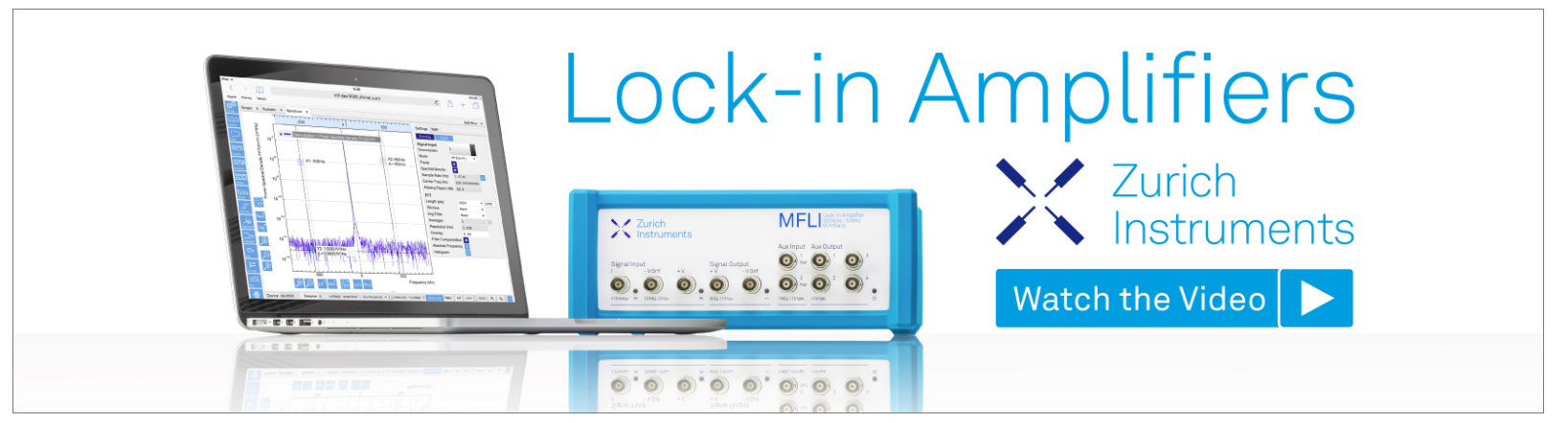




\title{
Polarized optical feedback from an extremely short external cavity for controlling and stabilizing the polarization of vertical cavity surface emitting lasers
}

\author{
Mikel Arizaleta Arteaga ${ }^{\text {a) }}$ \\ Department of Electrical and Electronic Engineering, Universidad Pública de Navarra, 31006 Pamplona, \\ Navarra, Spain \\ Olivier Parriaux \\ Laboratoire TSI, UMR CNRS 5516, 18 Rue B. Lauras, F-42000 Saint-Etienne, France \\ Manuel López-Amo \\ Department of Electrical and Electronic Engineering, Universidad Pública de Navarra, 31006 Pamplona, \\ Navarra, Spain \\ Hugo Thienpont and Krassimir Panajotov ${ }^{\text {b) }}$ \\ Department of Applied Physics and Photonics, Vrije Universiteit Brussel, 1050 Brussels, Belgium
}

(Received 26 January 2007; accepted 12 February 2007; published online 19 March 2007)

\begin{abstract}
The authors present experimental results showing that polarization selective optical feedback from an extremely short external cavity induces switching between the two fundamental transverse modes with orthogonal linear polarization of the otherwise (without optical feedback) polarization stable vertical cavity surface emitting laser. Moreover, when properly selecting the external cavity length within a subwavelength range the polarization state of the emitted light can be chosen in either of the two fundamental transverse modes and stabilized against injection current variations. The numerical results reported are in good agreement with experiments. (C) 2007 American Institute of Physics. [DOI: 10.1063/1.2714301]
\end{abstract}

Their inherent performance advantages over the conventional edge emitting lasers, such circular beam shape, twodimensional array fabrication, and high speed of operation, have made the vertical cavity surface emitting lasers (VCSELs) very desired laser sources for a broad range of applications. However, VCSELs suffer from a nonstable polarization. Small, unpredictable anisotropies introduced during the manufacturing process make VCSELs have two preferred orthogonal polarization directions of the fundamental transverse mode. ${ }^{1,2}$ The net gain difference between these two orthogonal linearly polarized (LP) modes is small and polarization switching (PS) can happen when changing the injection current and/or the temperature of operation of the device. $^{3,4}$ In order to avoid such uncertainty in VCSEL polarization many different techniques based on introducing polarization dependent modal gain and/or loss have been proposed so far (see, for example, Refs. 5-7). With such techniques the VCSEL polarization is fixed during fabrication and cannot be changed afterwards. On the other hand, polarized and isotropic optical feedbacks from short and long external cavities have been proposed to achieve active polarization control of VCSELs. ${ }^{8-11}$ Even polarization stabilization has been shown in the case of high strength polarized optical feedback. ${ }^{12-14}$ Such a technique allows to actively control the VCSEL polarization but it cannot be implemented monolithically and the feedback strength required may lead to laser instabilities. In order to overcome those drawbacks, research has been driven towards much smaller

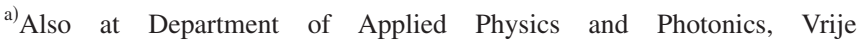
Universiteit Brussel, 1050 Brussels, Belgium; electronic mail: mikel.arizaleta@unavarra.es

b) Also at Institute of Solid State Physics, 1784 Sofia, Bulgaria.
}

external cavities of a few tenths of a micrometer. Recently, polarization active control and stabilization of VCSELs have been achieved using an isotropic optical feedback from an extremely short external cavity (ESEC), ${ }^{15,16}$ turning to be a very generic approach. ${ }^{17}$ Theoretically, it has also been predicted that the impact of a polarization selective optical feedback from an ESEC on the VCSEL polarization would be much stronger. ${ }^{18}$ However, experimental evidences have not been presented so far. In this letter we present experimental results showing how polarized optical feedback from an ESEC affects the polarization of the VCSELs. We prove that polarization active control and stabilization of VCSELs is possible by selecting the external cavity length. A very good agreement between the experimental results and the numerical simulations carried out using the model presented in Ref. 18 is also demonstrated.

In the experiments we use an oxide-confined GaAs/InGaAs quantum well VCSEL emitting at $1.005 \mu \mathrm{m}$. The solitary VCSEL (without optical feedback) emits in its fundamental transverse mode up to 1.75 times the threshold current. Although no special measures were taken to introduce polarization dependent gain/losses in the VCSEL, the polarization of the emitted light is measured to be linear and stable against injection current variations within the current operation range of the device. A more complete description of the laser is presented elsewhere. ${ }^{16}$ The ESEC is made by placing a single mode optical fiber at a few micrometers from the VCSEL with a polarization dependent mirror glued at its front face with an index matching glue. The polarization dependent mirror is a subwavelength grating made of $\mathrm{SiO}_{2}$ and $\mathrm{Si}_{3} \mathrm{~N}_{4}$. The spectral dependence of its reflectivity is shown in Fig. 1. At the wavelength of operation of the VCSEL $(1005 \mathrm{~nm})$ the reflectivity of the grating is measured at 


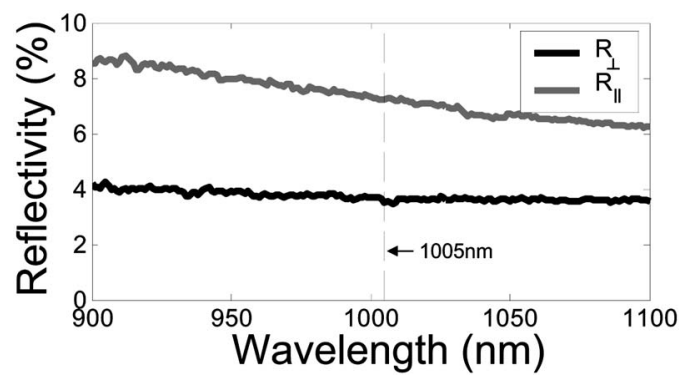

FIG. 1. Experimentally measured reflectivity spectrum for light linearly polarized parallel to the grating grooves $\left(R_{\|}\right)$and perpendicular to them $\left(R_{\perp}\right)$.

a normal incidence to be $R_{\|}=7.25 \%$ and $R_{\perp}=3.55 \%$ for linear polarization parallel and perpendicular to the direction of the grating, respectively. In order to allow for a precise alignment, the fiber and the VCSEL are mounted on a five axis translation stage and on a three axis piezoelectric controlled translation stage, respectively. The external cavity (EC) length is varied by moving the VCSEL along the direction of the light emission by means of one of the piezocomponents of its stage in steps of $20 \mathrm{~nm}$ and with a precision of $5 \mathrm{~nm}$. The EC lengths reported in the following are relative- the zero corresponds to an EC length smaller than $20 \mu \mathrm{m} .{ }^{16}$ The light emitted by the laser is coupled into the single mode optical fiber through the polarization dependent mirror. This allows measuring of the polarization resolved optical power at the far end of the fiber with one beam splitter, two polarizers, and two photodetectors. The far end of the fiber is ended in an FC-APC connector to avoid feedback from the fiber-air interface. An all fiber polarization controller is used in order to compensate any possible unintentional birefringence introduced in the fiber.

As we have already mentioned VCSELs emit in two orthogonal LP fundamental transverse modes. Due to the birefringence of the laser cavity, these two LP modes have slightly different frequencies and are, therefore, referred to in the rest of the letter as the high and low frequency LP modes. First, we orient the axes of the subwavelength grating at $45^{\circ}$ from the axes of these two LP modes. We decrease the EC length in steps of $20 \mathrm{~nm}$ and at each EC length we vary the injected current (from 2 to $12 \mathrm{~mA}$ and back to $2 \mathrm{~mA}$ in steps of $0.1 \mathrm{~mA}$ ) and measure the polarization resolved power versus current $(P I)$ curves. The impact of the optical feedback from the grating on the polarization properties of the VCSEL can be observed by mapping the polarization of emission on the plane EC length-injection current [see Fig. 2(a)]. As can be observed from Fig. 2(a), the emitted polarization does not depend on the EC length and the VCSEL exhibits the same polarization characteristics as in the solitary case (high frequency mode stable independently of the injection current). The only dependence on the EC length in the mapping is the modulation of the threshold current with half of the wavelength of operation of the VCSEL. ${ }^{19}$ Such a mapping resembles the case of optical feedback from a low reflectivity polarization insensitive mirror. ${ }^{17}$ Indeed, for a grating orientation of $45^{\circ}$ the mirror reflectivity seen by the high frequency mode $\left(R_{H}\right)$ and the one seen by the low frequency mode $\left(R_{L}\right)$ are equal. Therefore, the measured mapping is similar to the one obtained with a polarization insensitive mirror of a similar reflectivity [compare Figs. 2(a) and 2(b)].

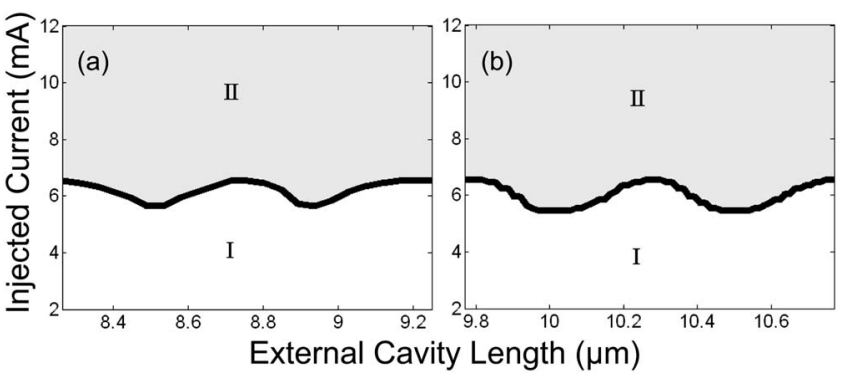

FIG. 2. Experimentally obtained mapping of the polarization states of a VCSEL subject to optical feedback from an ESEC. (a) External mirror is a diffraction grating with axes oriented at $45^{\circ}$ with respect to the axes of the VCSEL LP states. (b) Cleaved facet of the fiber [external mirror reflectivity of $\left.4 \%\left(R_{H}=R_{L}=4 \%\right)\right]$. The thick black line represents the threshold current below which the laser is not emitting (zone I). In zone II the VCSEL is stable and emits in its high frequency mode.

We now align the axes of the grating with the ones of the VCSEL LP modes in such a way that the high frequency mode polarization is parallel to the direction of the grating $\left(R_{H}=R_{\|}=7.25 \%\right.$ and $\left.R_{L}=R_{\perp}=3.55 \%\right)$. We sweep again the EC length and the injection current and map the polarization state [see Fig. 3(a)]. As it can be seen from this figure, the threshold current is modulated again with a period equal to half of the wavelength of operation of the VCSEL, but the amplitude of such a modulation is, however, larger than in the previous situation [see Fig. 2(a)]. Within each period of modulation and selecting the EC length we can observe three different polarization resolved power versus current curves. The VCSEL can show stable polarization operation either in its high [cut A in Fig. 3(a)] or its low [cut B in Fig. 3(a)] frequency mode at any injection current or it can exhibit polarization switching through a hysteresis region [cut $\mathrm{C}$ in Fig. 3(a)]. The current at which PS occurs can be widely tuned by selecting the EC length. Therefore, both a polarization control and a polarization stabilization of VCSELs can be achieved by means of polarization dependent feedback from an ESEC. The selection of the stable polarization mode is performed by choosing the EC length.

Now, we orient the axis of the grating at $90^{\circ}$ with respect to the axes of the VCSEL LP modes, i.e., the low frequency mode polarization is now parallel to the direction of the grating $\left(R_{L}=R_{\|}=7.25 \%\right.$ and $\left.R_{H}=R_{\perp}=3.55 \%\right)$. With such a con-

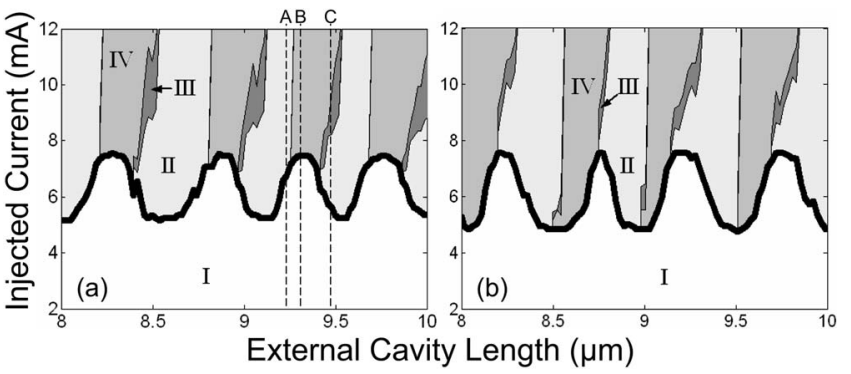

FIG. 3. Experimentally obtained mapping of the polarization states of a VCSEL subject to polarization sensitive optical feedback from an ESEC. The orientation of the grating grooves is parallel to the (a) high frequency mode polarization direction $\left(R_{H}=R_{\|}=7.25 \%\right.$ and $\left.R_{L}=R_{\perp}=3.55 \%\right)$ and (b) low frequency mode polarization direction $\left(R_{L}=R_{\|}=7.25 \%\right.$ and $R_{H}=R_{\perp}$ $=3.55 \%)$. A grayscale is used to distinguish between the different zones. The darkest gray zone (zone III) between zones II and IV represents the bistable region. The thick black line represents the threshold current below which the laser is not emitting (zone I). In zone II (IV) the VCSEL is stable and emits in its high (low) frequency mode. 


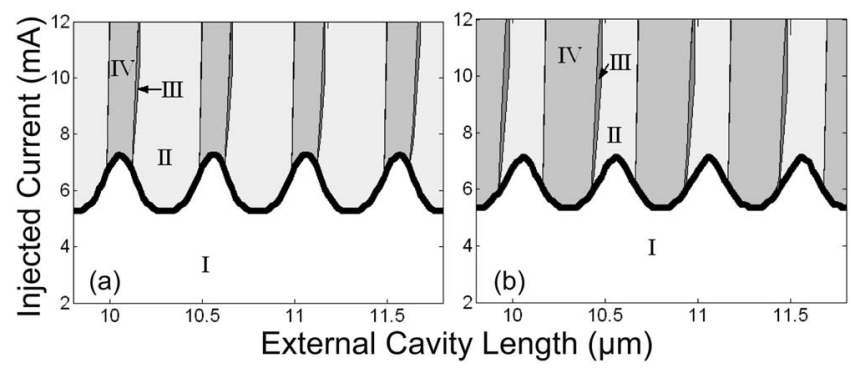

FIG. 4. Numerically obtained mapping of the polarization states of a VCSEL subject to polarization sensitive optical feedback from an ESEC. The external mirror reflectivity is set to (a) $R_{H}=7.25 \%$ and $R_{L}=3.55 \%$ and (b) $R_{H}=3.55 \%$ and $R_{L}=7.25 \%$. A grayscale is used to distinguish between the different zones in the same way as in Fig. 3.

figuration both the polarization stabilization and the polarization control are again possible. The mapping of the polarization states shows again regions of EC length at which either the high or the low frequency mode is stable against current variations [see Fig. 3(b)]. However, the location of such stable regions has changed within each period of modulation of the threshold current with respect to the previous grating orientation [compare Figs. 3(a) and 3(b)].

We numerically simulate the ESEC-VCSEL system using a two mode rate equation model presented in Ref. 18. The parameters and their meanings are given in Ref. 18 except for the following: $n_{0}^{H(L)}=3.3916(3.39195), d n / d J=2.7$ $\times 10^{-3} \mathrm{~mA}^{-1}, \quad g_{H(L)}=2.17(2.118) \times 10^{-8} \mu \mathrm{m}^{2}, \quad d g_{H(L)} / d J=$ $-9(-8.6) \times 10^{-10} \mu \mathrm{m}^{2} \mathrm{~mA}^{-1}, \quad s=3 \mu \mathrm{m}, \quad L_{c}=1.7695 \mu \mathrm{m}$, $\varepsilon_{s(c)}=2.5(5) \times 10^{-5} \mu \mathrm{m}^{3}$, and $r_{2}=0.9838$, which are chosen to fit the solitary VCSEL PI curve. ${ }^{16}$ Figure 4 shows the numerically obtained mapping of the polarization states for two different configurations of the external mirror reflectivities, demonstrating good agreement with the experimental results. Our model explains the polarization stabilization as a consequence of the loss difference between the LP modes introduced by the external polarization dependent mirror. Due to a change in the phase of the reflected field, the losses of each LP mode are periodically modulated when varying the EC length. ${ }^{15}$ The different mirror reflectivities for the two LP modes make the modulation amplitude of the losses different for the two modes. This leads to a very large amplitude modulation of the net gain difference between modes. Such a large net gain difference modulation allows for controlling the polarization of the VCSEL over the full range of injection current by slightly changing the EC length [for example, around $11 \mu \mathrm{m}$ in Fig. 4(a)]. The very thin hysteresis region observed within each threshold modulation period is not vertical because of the wavelength variation due to current induced self-heating of the laser.

Summarizing, we present experimental results showing that polarization sensitive optical feedback from an ESEC can induce switching between the two fundamental transverse modes with orthogonal linear polarization of the otherwise polarization stable VCSEL (without optical feedback). Moreover, by properly selecting the EC length within a subwavelength range we can stabilize the polarization of the light emitted by the VCSEL against injecting current variations in any of the two fundamental transverse modes. Our numerical results are in good agreement with the experiments. They reveal that the modulation of the difference of the losses between the two LP modes caused by the nonequal external mirror reflectivities is responsible for the strong polarization dependence on the EC length. The present external mirror polarization anisotropy (reflectivities of $7.25 \%$ and $3.55 \%$ for the two orthogonal polarizations) is enough to make polarization properties of the VCSEL depend mostly on the EC length than on the injecting current. Therefore, the polarization selective optical feedback is a very promising and generic technique to achieve active polarization control and stabilization of VCSELs. A monolithic device implementing such an idea is suggested in Ref. 20, namely, growing a second cavity on the VCSEL and etching a surface grating on top of it.

The authors acknowledge the financial support of the Public University of Navarra, of BELSPO IAP 6/10, of FWO-Vlaanderen, of OZR-VUB for the GOA and IOF projects, and Project No. TEC-2004-05936-C02-01 of MEC (Spain).

${ }^{1}$ C. J. Chang-Hasnain, J. P. Harbison, G. Hasnain, A. C. V. Lehmen, L. T. Florez, and N. G. Stoffel, IEEE J. Quantum Electron. 27, 1402 (1991).

${ }^{2}$ A. K. Jansen van Doorn, M. P. van Exter, and J. P. Woerdman, Appl. Phys. Lett. 69, 1041 (1996).

${ }^{3}$ K. D. Choquette, D. A. Richie, and R. E. Leibenguth, Appl. Phys. Lett. 64, 2062 (1994).

${ }^{4}$ B. Ryvkin, K. Panajotov, A. Georgievski, J. Danckaert, M. Peeters, G. Verschaffelt, H. Thienpont, and I. Veretennicoff, J. Opt. Soc. Am. B 16, 2106 (1999).

${ }^{5}$ D. Choquette and R. E. Leibenguth, IEEE Photonics Technol. Lett. 6, 40 (1994).

${ }^{6}$ P. Debernardi, J. M. Ostermann, M. Feneberg, C. Jalics, and R. Michalzik, IEEE J. Sel. Top. Quantum Electron. 11, 107 (2005).

${ }^{7}$ H. Uenohara, K. Tateno, T. Kagawa, Y. Ohiso, H. Tsuda, T. Kurokawa, and C. Amano, IEEE Photonics Technol. Lett. 11, 400 (1999).

${ }^{8}$ T. H. Russell and T. D. Milster, Appl. Phys. Lett. 70, 2520 (1997).

${ }^{9}$ P. Besnard, F. Robert, M. L. Chares, and G. Stephan, Phys. Rev. A 53, 3191 (1997).

${ }^{10}$ A. Valle, L. Pesquera, and K. A. Shore, IEEE Photonics Technol. Lett. 10, 639 (1998).

${ }^{11}$ M. Sciamanna, K. Panajotov, H. Thienpont, I. Veretennicoff, P. Megret, and M. Blondel, Opt. Lett. 28, 1543 (2003).

${ }^{12}$ S. J. Schablitsky, L. Zhuang, R. C. Shi, and S. Y. Chou, Appl. Phys. Lett. 69, 7 (1996).

${ }^{13}$ Y. Hong, P. S. Spencer, and K. A. Shore, Opt. Lett. 29, 2151 (2004).

${ }^{14}$ C. Masoller and M. S. Torre, IEEE J. Quantum Electron. 41, 483 (2005).

${ }^{15}$ K. Panajotov, M. Arizaleta, M. Camarena, H. J. Unold, J. M. Ostermann, R. Michalzik, and H. Thienpont, Appl. Phys. Lett. 84, 2763 (2004).

${ }^{16}$ M. Arizaleta Arteaga, M. López-Amo, H. Thienpont, and K. Panajotov, Appl. Phys. Lett. 89, 091102 (2006).

${ }^{17}$ M. Arizaleta Arteaga, M. López-Amo, H. Thienpont, and K. Panajotov, Appl. Phys. Lett. 90, 031117 (2007).

${ }^{18}$ M. Arizaleta Arteaga, H. J. Unold, J. M. Ostermann, R. Michalzik, H. Thienpont, and K. Panajotov, IEEE J. Quantum Electron. 42, 89 (2006).

${ }^{19}$ A. Hsu, J.-F. P. Seurin, S. L. Chuang, and K. D. Choquette, IEEE J. Quantum Electron. 37, 1643 (2001).

${ }^{20}$ M. Arizaleta Arteaga, H. Thienpont, and K. Panajotov, Patent No. PCT/ EP2006/005835 (20 June 2005). 